\title{
RETRACTED ARTICLE: Global existence and general decay estimates of solutions of the degenerate or non-degenerate Kirchhoff equation with general dissipation
}

\author{
Abbes Benaissa and Aissa Guesmia
}

The Article "Global existence and general decay estimates of solutions for degenerate or nondegenerate Kirchhoff equation with general dissipation" has been withdrawn for copyright reasons.

\author{
A. Benaissa \\ Laboratory of Mathematics, \\ Djillali Liabes University, \\ P. O. Box 89, Sidi Bel Abbes 22000, Algeria \\ E-mail: benaissa_abbes@yahoo.com \\ A. Guesmia \\ Laboratory of Mathematics and \\ Applications of Metz (LMAM), \\ Paul Verlaine - Metz University, \\ Bat. A, Ile du Saulcy, \\ 57045 Metz Cedex 01, France \\ E-mail: guesmia@univ-metz.fr
}

